\title{
Accurate Microwave High Power Measurements Using a Cascaded Coupler Method
}

\author{
Kenneth E. Bramall* \\ Institute for Basic Standards, National Bureau of Standards, Boulder, Colorado 80302 \\ The use of directional couplers to extend the range of low-level power meters is a well established \\ technique. Calibration of bolometer-coupler units up to $20 \mathrm{~dB}$ is routine at NBS and other laboratories \\ and allows power measurement to the $1 \mathrm{~W}$ level. For higher coupling ratios, however, the problems in \\ calibration using conventional techniques becomes more difficult. This paper describes a cascade \\ method which allows calibration of low power meter-coupler combinations for measurement up to the \\ megawatt range. \\ The technique is based upon system linearity and the uncertainty limits are shown to be 1 percent \\ to 1.5 percent using NBS low power standards. By using the NBS calibration services and commercially \\ available equipment, the above power levels can be measured with uncertainty limits less than 2.7 \\ percent. The method can also be adapted to measurement of large coupling ratios $(20-80 \mathrm{~dB})$. \\ Results are given for power (200 watts in WR-90 waveguide) and coupling ratio (40 dB coaxial \\ coupler) measurements.
}

Key words: Accurate; calibration; directional coupler; high power; measurement; microwave.

\section{Introduction}

High level (above $1 \mathrm{~W}$ ) microwave $\mathrm{CW}$ power measurements are commonly referenced to low-power standards by using a low-level power meter in combination with a power-reducing device such as a directional coupler or attenuator. Although better known and more economical than other methods for measuring high power, use of this method has been limited by uncertainties in relating the high power to the power indicated by the low-level power meter. An improved technique for determining this relationship is the main topic of this paper. The use of the directional coupler is emphasized, but the basic ideas presented will also apply to other attenuating or coupling devices.

\section{Cascaded Feed-Through Power Meter Calibration Technique}

The basic principles of the new calibration technique are described by discussing the calibration of a $40 \mathrm{~dB}$ coupler in combination with a low-level sidearm power meter having a dynamic range of $20 \mathrm{~dB}$ $(0.1$ to $10 \mathrm{~mW})$.

It has been shown that the best accuracy is obtained by calibrating the sidearm power meter and the coupler as a unit [1]. ${ }^{1}$ This requires a measurement of the coupler output power while a reading is obtained on its sidearm power meter. If a standard power meter

*Electromagnetics Division, Power-Current-Voltage Standards, National Bureau of Standards, Boulder, Colo. 80302.

${ }^{1}$ Figures in brackets indicate the literature references at the end of this paper. is available for measuring power in the range of 1 to $100 \mathrm{~W}$ (the power needed to obtain a reading within the range of the sidearm power meter) the calibration can be readily achieved. For the sake of explaining the technique, however, the calibration is to be achieved using a $10 \mathrm{~mW}$ standard power meter.

The calibration procedure employs an auxiliary 20 $\mathrm{dB}$ coupler and sidearm power meter with a dynamic range of $20 \mathrm{~dB}(0.1$ to $10 \mathrm{~mW})$ to transfer the calibration from the $10 \mathrm{~mW}$ standard power meter to the 40 $\mathrm{dB}$ coupler unit. Before starting the calibration, the 20 $\mathrm{dB}$ coupler is connected to the $40 \mathrm{~dB}$ coupler as shown in figure 1. At first glance, it appears that two coupler type, feed-through powermeters are in series. However, an alternate interpretation is also possible. In particular, the $40 \mathrm{~dB}$ coupler can be regarded as an extension of the main arm of the $20 \mathrm{~dB}$ coupler so that terminal

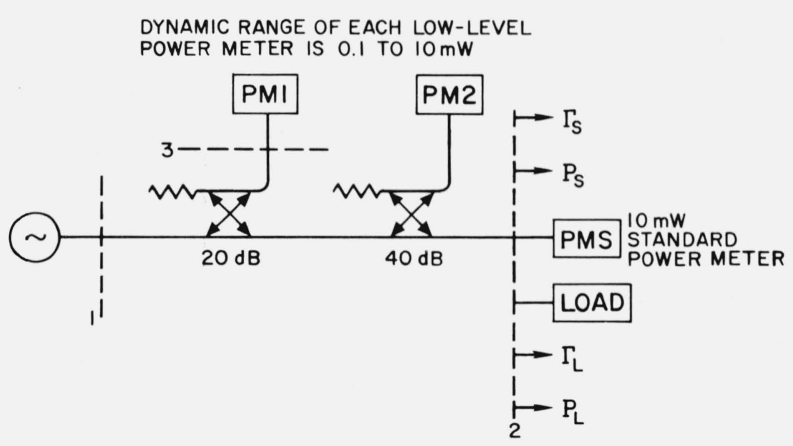

FIGURE 1. Basic cascaded coupler system. 
surface 2 serves a dual role; it is the output port of both the 20 and $40 \mathrm{~dB}$ couplers.

The $20 \mathrm{~dB}$ coupler unit (containing the $40 \mathrm{~dB}$ coupler unit) is calibrated by obtaining simultaneous readings on its sidearm power meter (PMl) and on the $10 \mathrm{~mW}$ standard power meter connected to terminal surface 2 . If the standard power meter is now removed and replaced by a high power load (and for the present it will be assumed that their impedances are equal) the $20 \mathrm{~dB}$ coupler unit will permit measurements of the load power in the range of $10 \mathrm{~mW}$ to $1 \mathrm{~W}$. The only requirement is that the component parameters be linear, i.e., independent of power level.

As the $1 \mathrm{~W}$ level is approached, the indicator on the $20 \mathrm{~dB}$ coupler reaches the upper limit of its dynamic range $(10 \mathrm{~mW})$ and the indicator on the $40 \mathrm{~dB}$ coupler comes into the lower region of its dynamic range $(0.1$ $\mathrm{mW}$ ). Since the load power can be accurately predicted from the power meter on the sidearm of the $20 \mathrm{~dB}$ coupler, the $40 \mathrm{~dB}$ coupler unit is calibrated by obtaining simultaneous readings on the two sidearm power meters. The calibration is thus transferred from the 20 to the $40 \mathrm{~dB}$ coupler unit without introducing uncertainties associated with mismatch, with flange losses, or with losses occurring within the couplers. Following this calibration, the input power can be further increased another $20 \mathrm{~dB}$ and load powers in the range of 1 to $100 \mathrm{~W}$ determined by the $40 \mathrm{~dB}$ coupler-sidearm power meter unit. Again, the components must be linear.

As a practical matter, the power meter on the sidearm of the $20 \mathrm{~dB}$ coupler may be damaged by excessive power when the load power is increased beyond $1 \mathrm{~W}$. This power meter overload problem is simply avoided by removing the $20 \mathrm{~dB}$ coupler after the $40 \mathrm{~dB}$ coupler unit is calibrated. The calibration of the $40 \mathrm{~dB}$ coupler unit is not affected by doing this because a directional coupler, like other three-port devices, has the property that the power split between the main and sidearms is independent of the source characteristics (see appendix).

The method described in the previous paragraphs can obviously be extended to systems employing any number of cascaded couplers. For example, figure 2 shows four coupler units where the calibration procedure is as follows:

1. Adjust power input so both PMl and PMS are within their dynamic ranges.

2. Obtain simultaneous readings of PM1 and PMS.

3. Replace PMS with the high power load.

4. Increase power until PM2 is in its operating range (but upper limit of PMl is not exceeded).

5. Obtain simultaneous readings of PM1 and PM2.

6. Remove PMl and the $20 \mathrm{~dB}$ coupler from the system.

7. Increase power until PM3 is in its operating range (but upper limit of PM2 is not exceeded). ${ }^{2}$

\footnotetext{
${ }^{2}$ Since coupler 1 was removed from the system, PM1 may be transferred to coupler 3 Likewise PM2 may be transferred to coupler 4. Thus, only two power meters are required for any number of cascaded couplers. Although errors may be introduced by this procedure, they are negligible for couplers having large coupling coefficients. For couplers having smal coupling coefficients, the error can be made negligibly small by using load isolators on their idearms or by initially terminating the sidearms with load having reflection coeffients approximately equal to the sidearm power meters.
}

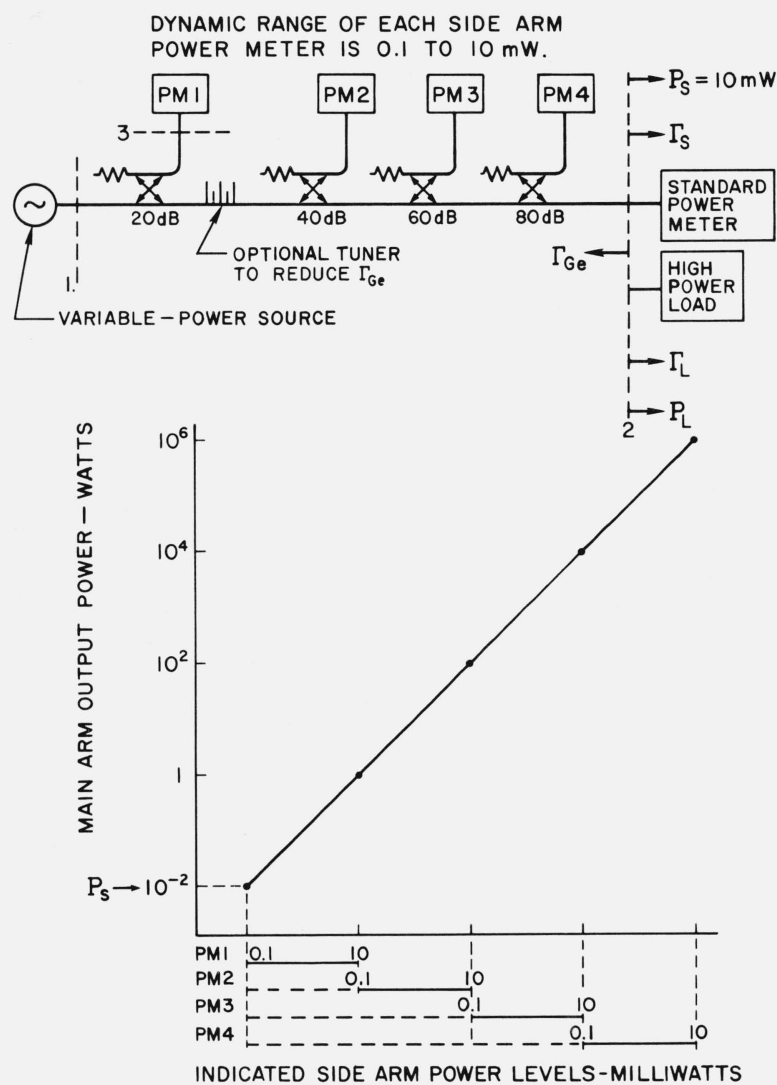

Figure 2. Cascaded coupler system used to calibrate an $80 \mathrm{~dB}$ coupler-power meter combination.

8. Obtain simultaneous readings of PM2 and PM3.

9. Remove PM2 and the $40 \mathrm{~dB}$ coupler from the system.

10. Increase power until PM4 is in its operating range (but upper limit of PM3 is not exceeded).

11. Obtain simultaneous readings of PM3 and PM4.

12. Remove PM3 and the $60 \mathrm{~dB}$ coupler from the system.

13. Measure power in the range $10^{4}$ to $10^{6} \mathrm{~W}$.

By following the steps above, the $80 \mathrm{~dB}$ coupler and its sidearm power meter are calibrated as a unit and the load power is given by the expression

$$
P_{L}=\frac{P_{1}^{\prime}}{P_{1}} \frac{P_{2}^{\prime}}{P_{2}} \frac{P_{3}^{\prime}}{P_{3}} \frac{P_{4}^{\prime}}{P_{4}} P_{s}
$$

where $P_{n}$ is the reading of the $n$th side arm power meter when a calibration was effected and $P_{n}^{\prime}$ is the reading of this power meter when it measured the load power. The reading of the standard power meter is $P_{s}$. This expression for the load power, $P_{L}$, is exact when the standard power meter and the load impedances are equal. The effects of dissimilar impedances will be considered next.

When the standard meter and the load impedances are not equal, mismatch is introduced when the former is exchanged for the latter. This is the only impedance 
mismatch introduced, however, because the same load is used for the remainder of the calibration procedure. The impedance mismatch introduced with the cascaded system is basically the same as for a single coupler unit and can be handled using existing techniques.

Engen [1] has shown that the load power determined by a single calibrated coupler unit is given by the expression

$$
P_{L}=\frac{P_{1}^{\prime}}{P_{1}} P_{s} \frac{1-\left|\Gamma_{L}\right|^{2}}{1-\left|\Gamma_{s}\right|^{2}}\left|\frac{1-\Gamma_{G E} \Gamma_{s}}{1-\Gamma_{G E} \Gamma_{L}}\right|^{2}
$$

where $P_{1}$ is the reading of the side arm power meter when the unit was calibrated with a standard power meter whose reading was $P_{s}$. The reading $P_{1}^{\prime}$ of the side arm power meter determines the power delivered to the load after it replaces the standard meter. The reflection coefficients of the standard meter and the load are given, respectively, by $\Gamma_{s}$ and $\Gamma_{L}$. The factor $\Gamma_{G E}$ is defined by Engen [2] as the equivalent generator reflection coefficient which the coupler provides, and its measurement is discussed in the referenced paper. This coefficient is given in terms of the coupler's scattering coefficients as

$$
\Gamma_{G E}=S_{22}-\frac{S_{21} S_{32}}{S_{31}}
$$

when the reference planes are chosen so that the source is connected to port 1 , the output of the coupler main arm is port 2 and the sidearm is port 3.

Similarly, an equivalent generator reflection coefficient $\Gamma_{G E}$ can be defined for the first feed-through power meter in the cascaded coupler system with reference planes as indicated in figure 2. By doing this and comparing the expression for the load power given in eq (1) with eq (2), eq (1) can be modified to include the effects of impedance mismatch. The results are:

$$
P_{L}=\frac{P_{1}^{\prime}}{P_{1}} \frac{P_{2}^{\prime}}{P_{2}} \frac{P_{3}^{\prime}}{P_{3}} \frac{P_{4}^{\prime}}{P_{4}} P_{S} \frac{1-\left|\Gamma_{L}\right|^{2}}{1-\left|\Gamma_{S}\right|^{2}}\left|\frac{1-\Gamma_{G E} \Gamma_{S}}{1-\Gamma_{G E} \Gamma_{L}}\right|^{2} .
$$

Only the $\Gamma_{G E}$ of the first feed-through power meter appears in eq (4), although an equivalent generator reflection coefficient can also be defined for each of the other feed-through power meters in the cascaded system. Unlike the first feed-through power meter, the others measure the power delivered to the same load that terminates their output when they are calibrated. As a result the power measurement is not affected by their equivalent generator reflection coefficients. They need not be known unless, as discussed below, the load is exchanged for another after the calibration is complete. The $\Gamma_{G E}$ of the first feedthrough power meter must be known, however, in order to evaluate the mismatch error in eq (4). This requires that $\Gamma_{G E}$ be measured before the calibration begins and the system is disassembled.

In addition to giving a method for measuring $\Gamma_{G E}$ in the above reference, Engen showed that it is possible to reduce the value of $\Gamma_{G E}$ for a single coupler by using a tuner in the output arm. A tuner can also be incorporated into the cascaded system and used to reduce the value of $\Gamma_{G E}$ that exists when the load replaces the standard meter. The tuner can be added anywhere between the first coupler and the output port. There is an advantage, however, in locating it between the first and second couplers. After the calibration is transferred to the second coupler, the tuner becomes part of the source for this coupler. Thus the tuner can be removed with the first coupler, eliminating the need for a tuner that will operate at high power levels.

An alternative method for measuring and reducing $\Gamma_{G E}$ is provided by employing the first coupler as part of a reflectometer $[3,4]$. When this reflectometer is tuned with respect to the output port, $\Gamma_{G E}$ is made small and a method is provided for measuring the reflection coefficient magnitudes of the standard meter and the load. Again, as with the single tuner, the added reflectometer components can be removed with the first coupler. More details about this method are given later with the experimental results.

The high power load can be exchanged for another after the calibration is complete. However, another mismatch error will be introduced. Specifically, if after the last coupler has been calibrated using a particular load, another load with a reflection coefficient $\Gamma_{L 2}$ is substituted, the expression for the power delivered to this second load is

$$
P_{L 2}=\frac{P_{1}^{\prime}}{P_{1}} \frac{P_{2}^{\prime}}{P_{2}} \frac{P_{3}^{\prime}}{P_{3}} \frac{P_{4}^{\prime}}{P_{4}} P_{s} \frac{1-\left|\Gamma_{L 2}\right|^{2}}{1-\left|\Gamma_{s}\right|^{2}}\left|\frac{1-\Gamma_{G E} \Gamma_{s}}{1-\Gamma_{G E} \Gamma_{L}}\right|^{2}\left|\frac{1-\Gamma_{G E}^{\prime} \Gamma_{L}}{1-\Gamma_{G E}^{\prime} \Gamma_{L 2}}\right|^{2}
$$

where $\Gamma_{G E}^{\prime}$ is the equivalent generator reflection coefficient associated with the last coupler, and the other terms are as previously defined. Evaluation of the additional mismatch error in eq (5) requires additional measurements to determine $\Gamma_{G E}^{\prime}$ and $\Gamma_{L 2}$.

\section{Application to Attenuation Measurements}

The calibration technique as described provides a method for accurately determining the power delivered to a particular high power load. The technique does this by using several power meters with limited dynamic ranges to produce the same results as would be obtained using one power meter with a large dynamic range. This characteristic also makes the technique useful for other applications such as evaluating the linearity of detectors with large dynamic ranges or for measuring attenuation using the power ratio method of measurement.

The power ratio method for measuring attenuation requires measurements of the input and output powers of a two-port device. This method has been shown to be capable of high accuracy when using bolometric power meters for attenuation measurements up to 20 or $30 \mathrm{~dB}$ [5]. A wider range of attenuation can now be measured using a cascaded coupler system, since it permits a wider range of input power levels 
to be measured. For best accuracy, the output power can be measured with the same standard power meter used to calibrate the cascaded coupler system. The maximum attenuation that can be measured is limited, of course, by the component's ability to withstand the high power. This limitation, for several measurements, is not as severe as it may seen. For example, by using power meters with dynamic ranges of 0.1 to $10 \mathrm{~mW}$, $40 \mathrm{~dB}$ of attenuation can be measured with a source of $1 \mathrm{~W}, 50 \mathrm{~dB}$ with a source of $10 \mathrm{~W}$, and $60 \mathrm{~dB}$ with a source of $100 \mathrm{~W}$. Several components including directional couplers can tolerate these (and higher) power levels.

The measurement of a coupler coupling coefficient is an attenuation measurement between the input and the side arm when the output is terminated in a nonreflecting load. An $80 \mathrm{~dB}$ coupler, for example, requires an input power level of $10 \mathrm{kw}$ in order to obtain a reading of 0.1 milliwatt at the side arm. A measurement of this input power is provided by using a cascaded system of 20,40 , and $60 \mathrm{~dB}$ coupler units. Using this system and computing the coupling coefficient yields

$K=10 \log _{10} \frac{P_{1}^{\prime}}{P_{1}} \frac{P_{2}^{\prime}}{P_{2}} \frac{P_{3}^{\prime}}{P_{3}} \frac{P_{s}}{P_{s 2}}\left|\frac{1-\Gamma_{G E} \Gamma_{s}}{1-\Gamma_{G E} \Gamma_{C}}\right|^{2} \frac{1}{\left|1-S_{33} \Gamma_{s}\right|^{2}}$

where $S_{m n}$ are the scattering coefficients of the coupler and $\Gamma_{C}$ is the reflection coefficient at the input to the coupler with the nonreflecting high power load and standard power meter connected. The expression for this reflection coefficient is

$$
\Gamma_{c}=S_{11}+\frac{S_{13} S_{31} \Gamma_{s}}{1-S_{33} \Gamma_{s}}
$$

Note that since the ratio of $P_{s} / P_{s 2}$ is taken, where $P_{s 2}$ is the side arm power reading obtained with the standard meter, a calibrated standard power meter is not required for these measurements. Consequently, this meter can be incorporated with a tuner to obtain $\Gamma_{s} \simeq 0$ and reduce the mismatch error introduced. The advantage of tuning $\Gamma_{G E}=0$ at the low power level is apparent.

\section{Alternate Calibration Method}

The system shown in figure 3 , is an alternate calibration method which uses attenuators between the coupler sidearms and the power meters. Compared with the purely cascaded system, it is physically shorter and the power ratio calibration steps can have any value if the attenuators $\left(A_{1}\right.$ and $\left.A_{2}\right)$ are continuously variable. The utility of this method is that it allows couplers having relatively low coupling ratios to be transformed into ones having the equivalent of high coupling ratios. For example, a $40 \mathrm{~dB}$ coupler can be transformed to the equivalent of a 60 or $80 \mathrm{~dB}$ coupler.

The calibration procedure for this system is similar to that for the purely cascaded system, the difference being that the calibration is transferred back and forth between power meters 1 and 2 as the power level

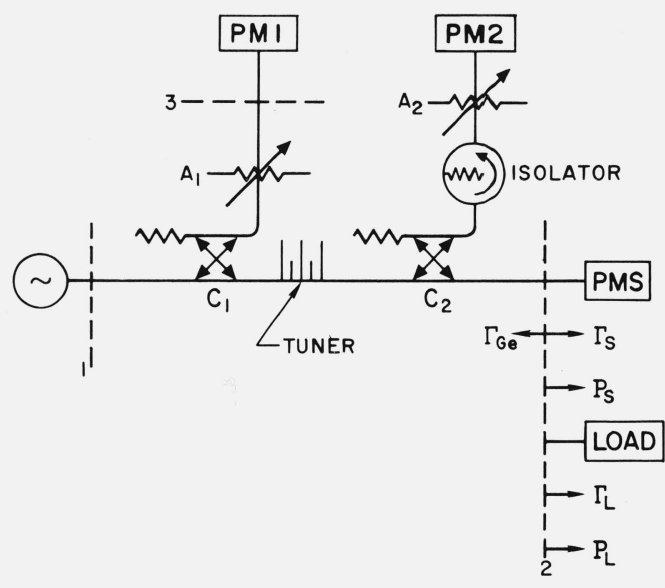

FIGURE 3. Variation of the cascaded coupler calibration technique. The attenuators are alternately used to reduce the coupling to one power meter while the other retains the calibration.

is increased. The attenuators are alternately used to reduce the coupling to one power meter while the other retains the calibration.

The first coupler unit can be considered as part of the source for the second unit and the setting of attenuator $A_{1}$ can be changed without introducing an error when the calibration is referenced to power meter 2. When the calibration is referred to power meter 1, however, a change in the setting of attenuator $A_{2}$ can introduce an error, because the amount of power reflected back into the system may change which in turn can change the relation between the first sidearm power meter reading and the load power. This error can be made negligibly small by using an isolator as shown in figure 3. The other errors associated with this calibration scheme are the same as for the strictly cascaded coupler system.

A tuner for reducing $\Gamma_{G E}$ can be inserted between the couplers. It can be removed after the calibration is first transferred to power meter 2 and an error will not be introduced when the calibration is transferred back to power meter 1. Similarly, a reflectometer can be used to reduce and evaluate the mismatch error introduced at arm 2.

\section{Experimental Systems and Results}

A WR-90 waveguide coupler system was assembled in the laboratory to obtain experimental data. The system incorporated a reflectometer and provided the capability of obtaining two separate calibrations of the last low power meter-coupler unit. This system is shown in figure 4 . One calibration for the $40 \mathrm{~dB}$ coupler unit was obtained by using couplers 1 through 4 to provide $10 \mathrm{~dB}$ power ratio calibration steps. The other calibration was obtained by using couplers 2 and 4 to provide power ratios of $20 \mathrm{~dB}$. The two calibrations disagreed by no more than 0.11 percent which indicates the precision of the method.

No unusual problems were encountered or unpredicted results obtained in performing the double 


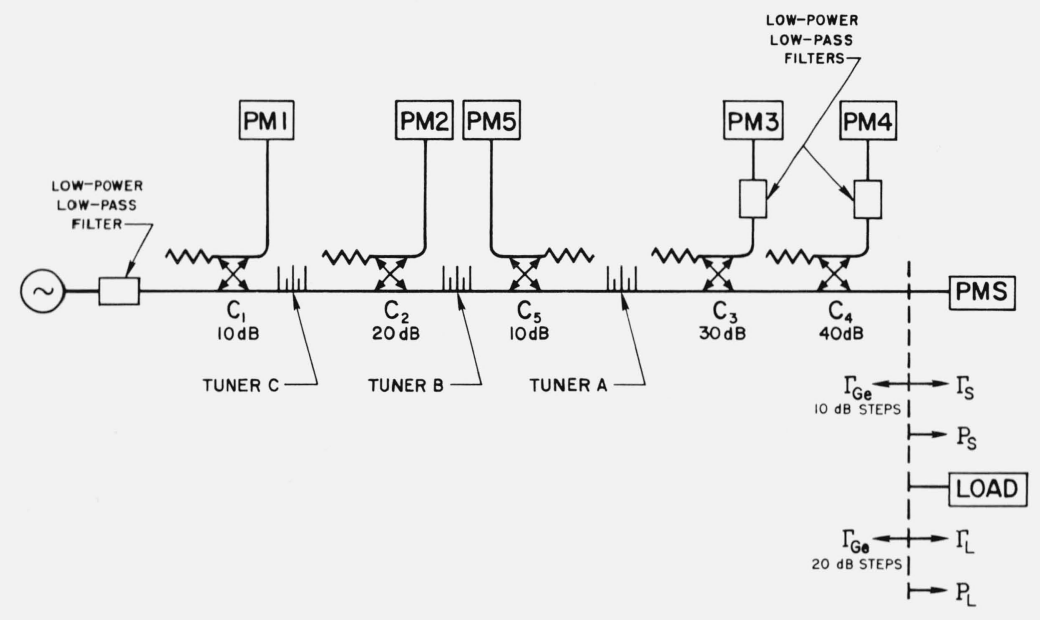

FIGURE 4. A coupler system that incorporates a reflectometer and provides the capability of obtaining two separate calibrations of the $40 \mathrm{~dB}$ coupler.

calibration. Intercomparison power measurements were made at the 70 to $200 \mathrm{~W}$ level between the 40 $\mathrm{dB}$ coupler unit and a stirred-water calorimeter [6]. The disagreement was around 1 percent, well within the combined error limit for the two meters.

In the tuning procedure for this system, tuner A was first adjusted so that the coupler sampling the reflected power (coupler 5) would have near infinite directivity. Tuner $B$ was then adjusted to obtain $\Gamma_{G E} \simeq 0$ for the system using power ratios of $20 \mathrm{~dB}$. Finally, tuner $\mathrm{C}$ was adjusted to obtain $\Gamma_{G E} \simeq 0$ for the system using power ratios of $10 \mathrm{~dB}$. All of the tuning was done with respect to the output of the system by using well known tuning procedures $[3,4]$. The tuning operation was performed by sliding high and low calibrated reflecting loads within a waveguide section attached as part of the coupler system. The low reflection load was used as the reflection coefficient standard. By using this approach, the reflectometer measured the combined reflection coefficient of the load and flange at the output of the system.

The reflectometer, the first two couplers, the three tuners, and the low-power low-pass filter in the main line were removed when the calibration was transferred to couplers 3 and 4.

A second system using coaxial components was assembled to measure the output to sidearm power ratios of a set of 5 coaxial couplers having nominal coupling values of $12,20,23,30$, and $40 \mathrm{~dB}$. These are slot type, quarter wave precision couplers as described by Hudson [7] and have $50 \mathrm{~dB}$ directivity and maximum VSWR of 1.02 at all ports. Their center frequency is $1.45 \mathrm{GHz}$ and measurements were made at frequencies in the range 1 to $2 \mathrm{GHz}$. For the lower value couplers (up to $20 \mathrm{~dB}$ ), the output-to-sidearm power ratio was measured directly using standard bolometric power meters $(\mathrm{VSWR}<1.03)$. The cascade system was used to measure the higher value couplers, and because VSWR of the ports is low, tuners were not required to reduce the value of $\Gamma_{G E}$. The limit of uncertainty in the measurement of the $40 \mathrm{~dB}$ coupler was computed to be 0.8 percent $(0.035 \mathrm{~dB})$ which includes the effects of impedance mismatch, repeatability, etc. The $40 \mathrm{~dB}$ coupler was also measured using the NBS coaxial calorimeter $[8]$ and a standard bolometric power meter. The two measured power ratio values differed less than 1 percent.

\section{Errors}

The limit of uncertainty in a high power measurement, using the cascade system, depends primarily upon the uncertainties introduced by the reflection coefficients and by the low level standard and side arm power meters. Since bolometric power meters conveniently yield accurate measurements in the 0.1 to $10 \mathrm{~mW}$ range, they are used in the following discussion to demonstrate the accuracy obtainable.

There are at least two methods available for obtaining less than 0.1 percent for the uncertainty introduced by the reflection coefficients. In one, the uncertainty is eliminated, in principle, by adjusting the reflection coefficients of the standard meter and the high power load to be equal. In the other method, the uncertainty is reduced by using a tuner to obtain a small, near zero, equivalent generator reflection coefficient and then correcting the measured power using the measured reflection coefficient magnitudes of the standard meter and the load.

The limit of uncertainty associated with the measurement of $P_{s}$ results primarily from the uncertainty in the bolometer mount effective efficiency and from the uncertainty in the measurement of the bolometrically substituted dc power. The National Bureau of Standards presently provides routine effective efficiency calibrations with limit of uncertainty of 1 percent for waveguide bolometer units [9]. The uncertainty can be as low as 0.2 percent, however, for a WR-90 waveguide mount calibrated against the NBS microcalorimeter [10].

Self-balancing bolometer bridges have been developed that enable a measurement of the substituted de power to be made with an uncertainty of 0.09 percent $+0.1 \mu \mathrm{W}$ [11]. This corresponds to uncertainties 
of $0.09,0.1$, and 0.19 percent in measurements of 10 , 1 and $0.1 \mathrm{~mW}$, respectively.

In a power ratio measurement, some of the systematic errors, such as those resulting from the bolometer mount reflection coefficient and its effective efficiency, cancel. The only uncertainties that need to be evaluated are those associated with the measurements of the bolometrically substituted de powers. By using the bolometer bridge mentioned above, power ratios of 10 and 100 can be measured with uncertainties of 0.19 and 0.28 percent, respectively.

The uncertainty in a high power measurement is the sum of the uncertainties introduced by the standard power measurement, by the mismatch error, and by the uncertainties introduced in the power ratio measurements. Table 1 shows an example breakdown of these uncertainties.

The power ratio uncertainties in table 1 can be re-

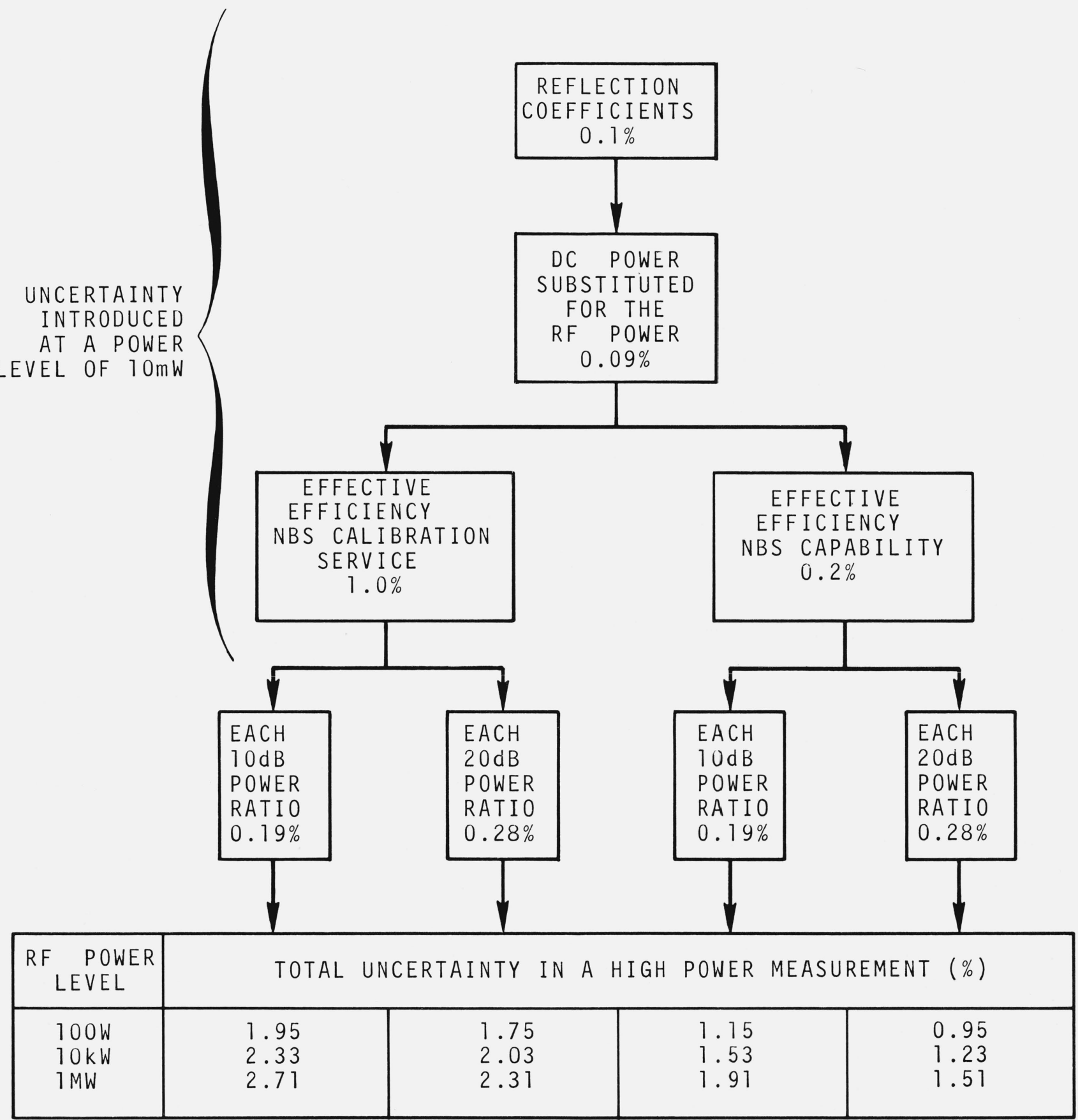

TABLE 1. Examples showing the uncertainty limit obtainable in a high power measurement by using the cascaded coupler calibration technique and equipment that is available commercially. 
duced by the use of the same bolometer bridge and accessory instrumentation in measuring the numerator and denominator powers. The amount of reduction will, in part, depend upon the systematic error common to both measurements.

Other sources of error that need to be considered in practice include rf power instability and the effects of bolometer mount temperature changes. Because the calibration is performed by making simultaneous power measurements, only short term stabilities are important, and the exacting power and temperature stability requirements are reduced.

The output from some high-power sources may contain large harmonic components. Since the coupling for the harmonic power is generally not the same as for the fundamental power, this can be a source of error. This error is easily reduced by incorporating low-power, low-pass filters with the power meters, as shown, for example, in figure 4.

In general, the calibration technique is based upon the assumption that the system is linear. The parameters of all the components used in the system must be independent of the rf power level and any other variable, such as environmental temperature, that may affect the linearity. The linearity of all the components except the directional couplers can be easily measured, since they operate at low power levels. The linearity of a coupler unit, however, must be measured at high power levels. Since this measurement requires a high power meter, another coupler unit can be used. In this way the power linearity of different types of couplers can be compared by comparing the side arm power readings as the power level is changed. With several comparisons any nonlinear components can be detected.

\section{Summary}

Accurate measurements of microwave power at high CW levels can be accomplished using the cascaded feed-through power meter calibration technique. The accuracy is based upon existing low-level standards, system linearity, and the ability to accurately measure power ratios. The technique can be used at any frequency in the $\mathrm{rf}$ spectrum where directional couplers (or other suitable 3-port devices), low-level power meters, and low-level power standards are available. (See fig. 5.)

Alternative systems, which employ directional couplers and attenuators, provide an additional flexibility in the calibration of coupler-low-powermeter units. The use of feed-through devices other than directional couplers may be advantageous, especially at the lower frequencies where the waveguide components are large. Since absolute power measurements are not required for measuring power ratios, detectors other than power meters, such as a precision variable attenuator and detector combination or an IF detector, are possible alternatives. Both of these detectors, however, may be subject to additional errors cuased by impedance changes.
Large values of attenuation or coupling ratio can be accurately measured using the cascade technique, provided the components can withstand the high power required.

The author expresses his appreciation to Lyman Elwell for his encouragement and suggestions in this work and to Glenn Engen for his valuable criticism in the preparation of this report. Credit is due to Lloyd Hendricks and Edward Andrusko for their efforts in obtaining the calorimeter-coupler intercomparison measurements.

The development of this technique was supported by the Advanced Research Projects Agency under Project DEFENDER and was monitored by M. I. Witow under ARPA Order No. 515.

\section{Appendix}

The calibration technique uses one calibrated feed-through power meter to calibrate another, and since a feed-through power meter has an input and two outputs, the theory for 3-port junctions is applicable. The ratio of the powers at ports 2 and 3 of any linear 3-port junction, with a source connected to port 1 as shown in figure 5 , is given by [12]

$$
\frac{P_{2}}{P_{3}}=\left|\frac{S_{21}}{S_{31}}\right|^{2} \frac{1-\left|\Gamma_{2}\right|^{2}}{1-\left|\Gamma_{3}\right|^{2}}\left|\frac{1-\left(S_{33}-\frac{S_{31} S_{23}}{S_{21}}\right) \Gamma_{3}}{1-\left(S_{22}-\frac{S_{21} S_{32}}{S_{31}}\right) \Gamma_{2}}\right|^{2}
$$

where $S_{M N}$ are the scattering coefficients of the junction, and $\Gamma_{2}$ and $\Gamma_{3}$ are the reflection coefficients of the loads at ports 2 and 3 .

The expression shows that the ratio of $P_{2}$ to $P_{3}$ is independent of the source reflection coefficient, $\Gamma_{G}$. For a linear system, the reflection and scattering coefficients are not a function of the power level and the ratio is independent of the source amplitude. In general, the ratio, or the calibration of a feed-through power meter, is independent of the source characteristics, and an error is not introduced by changing the source at port 1 .

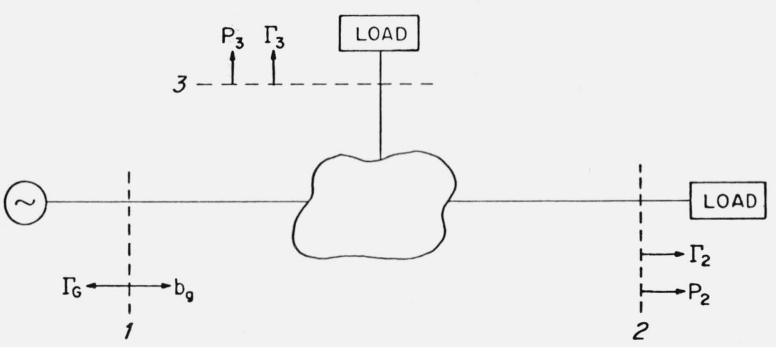

Figure 5. A generalized 3-port junction. 


\section{References}

[1] Engen, G. F., Recent developments in the field of microwave power measurements at the National Bureau of Standards, IRE Trans. on Instrumentation, vol. I-7, pp. 304-306, December 1958.

[2] Engen, G. F., Amplitude stabilization of a microwave signal source, IRE Trans. on Microwave Theory and Techniques, vol. MTT-6, pp. 202-206, April 1958.

[3] Engen, G. F., and Beatty, R. W., Microwave reflectometer techniques, IRE Trans. on Microwave Theory and Techniques, vol. MTT-7, pp. 351-355, July 1959.

[4] Anson, W. J., A guide to the use of the modified reflectometer technique of VSWR measurement, J. Research NBS, vol. 65C, pp. 217-223, October-December 1961.

[5] Engen, G. F., and Beatty, R. W., Microwave attenuation measurements with accuracies from 0.0001 to 0.06 decibels over a range of 0.01 to 50 decibels, J. Res. NBS, 64C, pp. 139-145, April-June 1960.

[6] Hendricks, L. J., A stirred-water calorimeter for the measurement of microwave power, to be published.

[7] Hudson, P. A., A high directivity, broadband coaxial coupler,
IEEE Trans. on Microwave Theory and Techniques, vol. MTT-14, pp. 293-294, 1966.

[8] Crawford, M. L., and Hudson, P. A., A dual-load flow calorimeter for $\mathrm{RF}$ power measurements to $4 \mathrm{GHz}$, J. Research NBS, vol. 71C, no. 2, pp. 111-117, April-June 1967.

[9] Rumfelt, A. Y., and Elwell, L. B., Radio frequency power measurements, Proc. IEEE, vol. 55, no. 6, pp. 837-850, June 1967

[10] Engen, G. F., A refined X-band microwave microcalorimeter, J. Research NBS, vol. 63C, pp. 77-82, July-September 1959.

[11] Engen, G. F., A self-balancing direct-current bridge for accurate bolometric power measurements, J. Research NBS, vol. 59, pp. 101-105, August 1957.

[12] Beatty, R. W., and Macpherson, A. C., Mismatch errors in microwave power measurements, Proc. IRE, vol. 41, pp. 1112-1119, September 1953.

[13] Kerns, D. M., and Beatty, R. W., Basic theory of waveguide junctions and introductory microwave network analysis, New York: Pergamon, 1967.

(Paper 75C3\&4-325) 